\title{
Small Intestine Necrosis Presented as acute Abdomen in Elderly Diabetic Type 2 Patient.
}

\author{
Aleksandar Mihail Kishman ${ }^{1}{ }^{*}$, Jovana Stanoeva ${ }^{1}$, Aleksandra Pejovska Josev ${ }^{2}$
}

Received: 20 October 2021 / Accepted: 15 November 2021 / Published online: 20 January 2022

This article is published with open access at https://journal.astes.org.al

(C) The author(s) 2022. \& Copyright (C) 2022, the Albanian Society for Trauma and Emergency Surgery

(c) The Albanian Journal of Trauma and Emergency Surgery is an Open Access Journal. All articles are distributed under the terms of the Creative Commons Attribution Non-Commercial License: http://creativecommons.org/licenses/by-nc/4.0/) which permits unrestricted non-commercial use, distribution, and reproduction in any medium provided the original work is properly cited.

\begin{abstract}
Background: Bowel infarction or gangrenous bowel represents an irreversible injury to the intestine resulting from insufficient blood flow. It is considered a medical emergency because it can quickly result in life-treating infection and death. Untreated bowel infarction quickly leads to life-threatening infection and sepsis and may be fatal. The only treatment for bowel infarction is immediate surgical repair and removal of the dead bowel segment. CT scan can help in the diagnosis, but CT angiography is most accurate in its ability to define the lesion. Diabetes mellitus is metabolic disease which presented with lack of insulin secretion (type $1 \mathrm{DM}$ ) or defect of insulin effect on tissues (type $2 \mathrm{DM}$ ) and both conditions lead to excess sugar in blood. DM can lead to serious medical conditions, which seen often in emergency department presented as acute complications diabetic ketoacidosis/hyperglycemic hyperosmolar condition (DKA/HHC) or acute conditions based on chronic complications. Uncontrolled diabetes mellitus especially in elderly patients with many years on antidiabetic therapy and other comorbidities can cause serious life treating illness and lead to death.

Case report: We present case of small intestine necrosis in 78-year-old women with hetero anamnesis for one-week abdominal pain and altered consciousness. The patient is many years diabetic with oral antidiabetic therapy and last four years with Insulin. She was diagnosed with acute abdomen condition and DKA/HHC. Initial glycemic values on admission were measured 38.87mmol/1. CT scan was made. She received emergency treatment for the current hyperglycemic disorder and after stabilization of primary condition she was transfer to operating room where laparotomy was performed. Resection of gangrenous small intestine was made and then continued postoperative care in ICU.
\end{abstract}

Conclusion: The case illustrates two emergency conditions in one time in poor regulated DM in elderly patient and possibility of acute presentation on chronic complication of DM.

Keywords: Small intestine necrosis, bowel infarction, Diabetic ketoacidosis, Hyperglycemic hyperosmolar condition, DM complication, acute abdomen, elderly population.

\section{Abbreviations:}

CT-Computed Tomography; DM-Diabetes Mellitus; DKA/ HHC-Diabetic Ketoacidosis/Hyperglycemic Hyperosmolar Condition; ICU-Intensive Care Unit; Le-Leucocytes; Ne-Neutrophils; Er- Erythrocytes; HB- Hemoglobin; CRP-C Reactive Protein; LDH-Lactate Dehydrogenase;

Original article, no submission or publication in advance or in parallel

\footnotetext{
* Corresponding author:

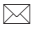

Aleksandar Mihail Kishman

1 University Clinic for Traumatology, Orthopedics, Anesthesiology, Reanimation, Intensive Care and Emergency Center, Skopje, Republic of North MACEDONIA

2 PHI Health Center of Skopje - Emergency Medical Service, Republic of North MACEDONIA
}

$C K-M B$ - Creatin Kinase- Muscle Brain; $C K$ - Creatine Kinase, AP- Alkaline Phosphatase, SaO2- Saturation; TA- Heart Pressure; HR- Heart Rate; AST-Aspartate Aminotransferase; ALT-Alanine Aminotransferase

\section{Introduction}

Diabetes mellitus is metabolic disease which presented with lack of insulin secretion (type $1 \mathrm{DM}$ ) or defect of insulin effect on tissues (type $2 \mathrm{DM}$ ) and both conditions lead to excess sugar in blood. DM can lead to serious medical conditions, which seen often in emergency department presented as acute complications DKA/HHC or acute conditions based on chronic complications. Clinical appearing of symptoms, like acute and chronic DM complications, not depends only of blood sugar levels and $\mathrm{HbA} 1 \mathrm{C}$, and on a number of predisposing and other factors (length of illness, non- 
adherence of the treatment mode, comorbidities, infection, stress, operation, immunity and nutritive status etc.). Acute complications of DM are hyper and hypoglycemic crises. Hyperglycemic crises are diabetic ketoacidosis (DKA) and hyperglycemic hyperosmolar condition (HHC) or combination of both (DKA/HHC). DKA appears more often in children DM type1, but can also be found in DM type 2 (in $34 \%$ cases). HHC is generally more often shown in DM type 2 in elderly patients with another comorbidities. Mortality in HHC is much higher (20 times) then DKA, in the range of 5-20\%. Precipitating factors for occurrence of hyperglycemic crises can be inadequate insulin therapy, infections, medications, endocrine disorders or another intercurrent diseases. As to the rate of onset of clinical manifestations, HHC appears in few days to week, but for acute episode of DKA it takes much longer. Symptoms in poor regulated DM are presented in few days but metabolic changes, typically for ketoacidosis, usually appear in short time (less than 24 hours). DKA have clinical symptoms: thirst, polydipsia, polyuria, polyphagia, weakness, exhaustion, nausea, vomiting. On examination have dry skin, dyspnea, tachypnea, (Kussmail) breathing, lowered reflexes, smell of acetone in the breath, often diffuse abdominal pain with GI symptoms, disorder of consciousness varies from confusion to severe disturbance of consciousness (coma less than $10 \%$ ). In clinical presentation of HHC symptoms are similar as in DKA but more pronounced. Disturbances in cognitive, sensory and motor functions as well as disturbances of consciousness are more pronounced in HHC. Characteristic sign in HHC is extreme dehydration, and may have focal neurological signs or EPI seizures and without acetone smell in breath. Diagnostic is based on laboratory parameters and degree of consciousness. To summarize, common features DKA and HHC are hyperglycemic and dehydration (which more commonly in HHC), the more important difference are ketone bodies in blood and urine in DKA. The dehydration appears secondary due to osmotic diuresis and leads to disturbance in hydro electrolyte balance and loss of glucose and electrolytes ( $\mathrm{Na}, \mathrm{K}, \mathrm{Ca}, \mathrm{Mg}$, and $\mathrm{P}$ ). [1]

Bowel infarction or gangrenous bowel represents an irreversible injury to the intestine resulting from insufficient blood flow. It is considered a medical emergency because it can quickly result in life-treating infection and death. [2]. Any cause of bowel ischemia, the earlier reversible form of injury, may ultimately lead to infarction if uncorrected. The causes of bowel ischemia or infarction include primary vascular causes as mesenteric ischemia, and other causes of bowel obstruction caused by intestinal adhesions after abdominal surgeries or by chronic infections such as diverticulitis, hepatitis and inflammatory bowel disease. [3] Causes of disturbance of the normal blood flow through the small bowel wall can be divided into acute and chronic forms, with the main etiologies. Acute forms includes: 1) Occlusive mesenteric ischemia presented as superior mesenteric artery occlusion (2/3 of cases) or superior mesenteric vein occlusion (5-10\% of cases), 2) Non-occlusive mesenteric ischemia (e.g. shock bowel) presented in systemic hypotension, blunt abdominal trauma, medication-induced (vasoconstriction from digitalis, amphetamines, cocaine), 3)Small vessel involvement like in chemotherapy-induced enteropathy, acute radiation enteritis or vasculitis (rare), 4) Small bowel obstruction -increasing bowel wall diameter increases bowel wall tension, which increases the resistance to incoming blood flow. Chronic etiology is seen in atherosclerotic stenosis of the superior mesenteric artery (e.g. mesenteric angina) and chronic radiation enteritis [4]

An infarcted or dead intestinal segment is a serious medical problem because intestines contain non-sterile fecal contents and high bacterial loads within the lumen. Progressive ischemia causes tissue breakdown and leads to bacteria spreading to the bloodstream. Untreated bowel infarction quickly leads to life-threatening infection and sepsis and may be fatal. The only treatment for bowel infarction is immediate surgical repair and removal of the dead bowel segment. [2]

Clinical, laboratory and radiology evaluations are used in diagnosis of acute complications of diabetes and acute abdominal pain. Altered mental state makes examination difficult because of not giving correct answers for the current medical condition. Laboratory results, D-dimers, CT scan and CT angiography are golden standard in diagnostic of bowel ischemia. Acknowledgment of characteristic bowel necrosis CT findings is crucial for determining the therapeutic attitude and the use of previous CT scans to compare the superior mesenteric artery diameter may help the radiologist to achieve an early diagnosis of nonocclusive mesenteric ischemia in an often critically ill patient population [5]

\section{Case report}

A 78-year-old woman was referred to our tertiary care institution- Emergency surgical center with complaints of abdominal pain, and because of present hypotension and altered mental status. She had a history of long-time diabetes type 2 insulin dependent and hypertension. From the regular therapy she had been taking antihypertensive therapy, oral antidiabetic and insulin therapy. In the morning her family member found her lying unconscious, in severe general condition and called emergency medical help. She was immediately taken to the internal emergency medicine center where she was primary examined and treated. On admission there she was conscious, disoriented, aware occasionally, passive position in bed. There were measured: glycaemia $30 \mathrm{mmol} / 1$, blood pressure $90 / 50 \mathrm{mmHg}$, saturation $92 \%$. ECG described as sinus tachycardia without heart suffering. She was tachypneic with abdominal breathing. On auscultation heard weakened vesicular breathing with crepitation in middle and basal structures of lungs bilateral. Palpation of abdomen showed soft abdomen at chest level with diffuse painful tenderness. From laboratory results: $\mathrm{Le}=14.710^{\wedge} 9 / \mathrm{L}$, $\mathrm{Ne}=94 \%, \quad \mathrm{CRP}=96.9 \mathrm{mg} / \mathrm{l}, \quad \mathrm{LDH}=269 \mathrm{U} / \mathrm{L}, \quad \mathrm{Ck}-\mathrm{MB}=45$, $\mathrm{CK}=939 \mathrm{U} / \mathrm{L}, \mathrm{AP}=157 \mathrm{U} / \mathrm{L}, \mathrm{Na}=134 \mathrm{mmol} / \mathrm{l}, \mathrm{K}=3.7 \mathrm{mmol} / \mathrm{l}$, 
acidum uricum=501umol/1, creatinine=197.6umol/1, urea $=11 \mathrm{mmol} / 1$, glucoza $=38.87 \mathrm{mmol} / 1$. Immediately was ordinated intra venous therapy with sol. $\mathrm{NaCl} 0.9 \% 500 \mathrm{ml}$, Insulin Actrapid 10 I.E., and amp. Urbazon $40 \mathrm{mg}$. There were made radiology evaluations (RTG on lungs and heart and abdomen- without particular finding. A consultation with a neurologist was performed, the examination showed no neurological suffering except necessary endocrinology treatment. A CT scan of brain was performed, and was showed age-appropriate brain parenchyma without focal parenchymal lesions or intra or extra axial fluid collection. Another consultation with Endocrinologist was performed. There were measured: blood pressure 140/80 $\mathrm{mmHg}$, $\mathrm{SaO} 2=92 \%$, glycemic $=32 \mathrm{mmol} / 1$, she was conscious, disoriented in space, time and persons. Therapy was applied Sol 0.9\% NaCl 500ml i.v + Insulin Fiasp 8 IE i.v + Insulin Fiasp 6 IE s.c Control glycaemia was $19 . .21 \mathrm{mmol} / \mathrm{l}$ and the patient were again transferred to Emergency internal center- Toxicology. Urinary catheter was inserted. Another laboratory result showed D-dimer $=39784.47 \mathrm{ng} / \mathrm{ml}$ and urine status: specific weight $=1.021, \mathrm{pH}=5.0$, protein $=30$, glucose $>=1000$, acetone $=15, \mathrm{Er}=8-10, \mathrm{Le}=10-15)$. Based on the result Amp. Clexane $60 \mathrm{mg}$ s.c. and Amp. Urbazon $40 \mathrm{mg}$ i.m. was given. Blood pressure $=90 / 60, \mathrm{SaO} 2=96 \%$, glycaemia $=15.7 \mathrm{mmol} / 1$. Because of the severe general condition and constant abdominal pain, she was transported to our Emergency surgery center. She was admitted somnolent, disoriented, with diffuse abdominal pain, distended abdominal wall, dyspneic, with peripheral cyanosis and low almost immeasurable blood pressure. Vital signs measured: blood pressure $85 / 37 \mathrm{mmHg}, \mathrm{SpO} 2=79 \%$, respirations $=40$ / $\min , \mathrm{HR}=135 / \mathrm{min}$. Immediately were placed 2 venous lines and ordinated Sol. Gelaspan 4\% $500 \mathrm{ml}$ i.v. + Sol. $\mathrm{NaCl} \mathrm{0.9 \%}$ $1000 \mathrm{ml}+$ Amp. Analgin i.v. and placed on oxygen support. Anesthesiologist and abdominal surgeon were called. After initial treatment parameters were $\mathrm{TA}=124 / 73 \mathrm{mmHg}$, $\mathrm{SaO} 2=98 \%$ (with O2), $\mathrm{HR}=120 / \mathrm{min}$. Blood analyses are made: $\mathrm{Er}=4.36 \quad 10^{\wedge} 12 / \mathrm{L}, \quad \mathrm{HB}=134 \mathrm{~g} / \mathrm{l}, \quad \mathrm{Le}=26.4$ $10^{\wedge} 9 / \mathrm{L}, \quad \mathrm{PLT}=155 \quad 10^{\wedge} 9 / \mathrm{L}, \quad \mathrm{CRP}=221 \mathrm{mg} / \mathrm{l}, \quad \mathrm{AP}=98 \mathrm{U} / \mathrm{L}$, amylase $=272.33 \mathrm{U} / \mathrm{L}, \quad \mathrm{AST}=114 \mathrm{U} / \mathrm{L}, \quad \mathrm{ALT}=33 \mathrm{U} / \mathrm{L}$, $\mathrm{LDH}=685 \mathrm{U} / \mathrm{L}, \quad \mathrm{Ca}=2.41 \mathrm{mmol} / \mathrm{l}, \quad \mathrm{Cl}=103 \mathrm{mmol} / \mathrm{l}$, $\mathrm{K}=4.5 \mathrm{mmol} / 1, \quad \mathrm{Na}=136 \mathrm{mmol} / 1, \quad$ bilirubin=7.4umol $/ 1$, creatinine $=213 \mathrm{umol} / 1, \quad$ s.urea $=11.9 \mathrm{mmol} / 1, \quad$ glycaemia $=$ $9.03 \mathrm{mmol} / \mathrm{l}$. CT scan on thorax and abdomen with contrast was performed. CT scan on thorax showed old peribronchititc changes basal on both sides on lungs. There are no signs of consolidation or pneumothorax. Basal pleura is thickened. Higher standing of diaphragmatic dome, free phrenic costal sinuses, without traumatic lesions. CT scan on abdomen with contrast showed presence of hiatal hernia, liver with homogeneous structure without focal changes, gallbladder with dense content. Spleen, pancreas, kidneys and adrenal regions are normal. Intestines are stagnant and dystended. Bladder without lesions with placed urinary catheter. No free fluid or enlarged lymph nodes are seen in abdomen or pelvic cavity. Skeleton with marked degenerative changes and skeletal demineralization.
She was diagnosed acute hyperglycemic metabolic disease ketoacidosis/hyperglycemic hyperosmolar condition. Abdominal surgeon diagnoses acute abdomen, suspected mesenteric occlusion/thrombosis. The patient was transferred to ICU to be reanimated and prepared for surgery. After stabilization of primary acute hyperglycemic condition, she was transferred to operating room where laparotomy was performed. Resection of gangrenous small intestine was made and then continued postoperative care in ICU.

\section{Discussion}

In these case report we present how acute hyperglycemic condition can be related with chronic complications followed by acute condition in poor regulated DM especially in elderly population. Diabetic patients may have chronic abdominal pain due to diabetes-related complications like neuritis, motor diseases of the gastrointestinal tract and autonomic dysfunction. Diabetic angiopathy is long term complication in poor regulate blood sugar. Vascular endothelium the primary defense against thrombosis, is abnormal in diabetes. Endothelial abnormalities undoubtedly play a role in the enhanced activation of platelets and clotting factors seen in diabetes. The fibrinolytic system, the primary means of removing clots, is relatively inhibited in diabetes due to abnormal clot structures that are more resistant to degradation and an increase in plasminogen activator inhibitor type 1(PAI-1) [6]. Atherosclerosis is 2-4 times more common in patients with diabetes and affects mainly carotid, coronary, iliac and lower limb arteries as well as aorta. Another less common complication is chronic mesenteric ischemia (CMI, intestinal angina), caused by atherosclerotic obstruction of the celiac artery and its branches and results in episodic of constant intestinal hypo perfusion or complete obstruction of the inferior mesenteric artery and cause intestinal gangrene [7].

Individuals with diabetes are in increased risk for acute mesenteric ischemia, according to study results published in Atherosclerosis. One population-based cohort study that utilizes data from medical claims databases in Taiwan investigate whether diabetes increases the risk of acute mesenteric ischemia (AMI). Using claims data from Taiwan's National health insurance program, 66,624 diabetic patients were enrolled from 1998 to 2009, and a comparison group of 266,496 individually matched subjects without diabetes was selected. The two groups were followed up until diagnosis of AMI, death, or the end of 2011. Incidence rates of AMI were assessed in both groups. The diabetes cohort had a higher incidence rate than the comparison cohort for AMI (0.56 vs.0.29 per 1,000 personyears). After adjusting for sex, age, comorbidity and health system utilization, the adjusted hazard ratio of diabetes was 1.32 (95\% confidence interval 1.11-1.56) for AMI. The risk of AMI associated with diabetes were greater in man (adjusted hazard ratio $=1.48,95 \%$ confidence interval 1.17 1.87) than in women (adjusted hazard ratio $=1.17,95 \%$ confidence interval 0.92-1.49). 
Conclusions of this study was that increased risk of AMI was occurred in patients with diabetes. Possibilities of this association being related with common set of risk factors in patients is not excluded. [8]

One case report showed ischemic jejunal structure developing after diabetic coma where ischemic structure of jejunum probably due to microaniopathy caused by diabetes mellitus was presented. The pathohistological finding showed ischemic enteritis with ulceration, granulation with microvascular proliferation, inflammatory cell infiltration, marked fibrosis, and hemosiderin deposition. Ketoacidosis increased blood viscosity and platelet aggregation, dehydration in addition to hyperglycemia, and occasionally results in occlusive or no-occlusive vascular disease of the intestine. Ischemic stricture of the bowel in the diabetic state is probably related to moderate ischemia resulting in ulceration and scar formation in the intestine. [9]

The clinical presentation of mesenteric ischemia and gangrene can vary with the underlying cause. Severe abdominal pain that is disproportionate to examination findings and that responds poorly to analgesia is a classic mode of presentation for acute mesenteric ischemia. [10] Although historically catheter angiography was the gold standard for imaging of suspected intestinal ischemia, CT has replaced it, with its ability to volumetrically assess the whole abdomen in multiple vascular phases, e.g., arterial, portal venous, and delayed. It can also have advantage of being able to diagnose alternative causes of acute abdominal pain. CT now is investigation of choice for patients with suspected intestinal ischemia. In general, it should be performed with intravenous contrast and a neutral luminal contrast (water). Although treatment will vary according to the severity and cause of ischemia, in general treatment is surgical by resection of necrotic tissue [10]

In this case because of the serious condition of ketoacidosis/hyperosmolar condition urgent treatment for lowering blood sugar levels should initially be implemented with isoosmolar solutions, insulin and electrolyte substitution according to medical condition and laboratory values. There should be given antibiotics and anticoagulant therapy because of high d-dimers. After appropriate resuscitation, normalizing vital signs and gaining stabile state operation for the acute abdominal condition should be the next step for life saving.

\section{Conclusion}

The incidence of intestinal ischemia and necrosis as acute abdominal condition is increasing because of uncontrolled diabetes mellitus. Especially elderly patients with many years on antidiabetic treatment and insulin dependency and other comorbidities, complications from the diabetic state on their organism often are worsened with time. Emergency complications like ketoacidosis and hyperosmolarity state can altered mental state and that lead to difficult examination. Beside of this life-threatening condition, in the same time acute presentation on chronic complication of DM can be presented. An infarcted or dead intestinal segment is a serious medical problem because of non-sterile fecal contents and high bacterial loads within the lumen Untreated bowel infarction quickly leads to life-threatening infection and sepsis and may be fatal because of the bacteria spreading to the bloodstream. [2] The only treatment for bowel infarction is immediate surgical repair and removal of the dead bowel segment. [10] Timely diagnosis and emergency medical treatment can prevent mortality.

\section{Declarations}

Funding: Not applicable

Competing interests: None declared

Contributors: All authors designed the manuscript. A. Kishman, J. Stanoeva and A.P.Josev acquired the data, which all authors analysed. All authors wrote the article, which all authors reviewed and approved for publication. All authors agreed to be accountable for all aspects of the work.

\section{References}

1. Initial treatment of urgent conditions in medicine. (2016) University of medicine faculty Belgrade, Nevena Kalezic

2. Vallicelli C, Coccolini F, Catena F, Ansaloni L, Montori G, Di Saverio S, Pinna AD (January 2011). Small bowel emergency surgery: literaturess review». World Journal of Emergency Surgery. 6 (1): 1. doi:10.1186/1749-7922-61. PMC 3025845. PMID 21214933

3. Belyaev O, Müller C, Uhl W (2009). Chapter 57: Small Bowel Obstructions. In Bland K (ed.). General Surgery: Principles and International Practice (2nd ed.). London: Springer. pp. 597-604. ISBN 978-1-84628-832-6. OCLC 314794037.

4. Dr Daniel J bell and Assoc Prof Frank Gaillard. Small bowel ischemia. Radiopaedia.org 2021

5. Carlos perez-Garcia, Enrique de Miguel Campos, Adriana Fernandez Gonzalo, carlos Malfaz. Jesus Javier Martin Pinacho. Carmen Fernandez Alvarez, Raguel Herranz Perez. Non-occlusive mesenteric ischaemia: CT findings, clinical outcomes and assessment of the diameter of the superior mesenteric artery. BRJ.Br J Radiol. 2018 Jan.91(1081)

6. Marcus E Carr.Diabets mellitus: A hypercoagulable state. ScienceDirect. ELSEVIER.Volume 15, Issue 1, JanuaryFebruary 2001 pages 44-54

7. George Panagoulias, Nicholas Tentolouris \&Spiros S Ladas Abdominal pain in an adult with Type 2 diabetes: A case report Cases Journal volume 1, Article number: 154 (2008)

8. Yen-Wei Chiu, Chi-Shin Wu, Pei-Chun Chen, Yu-Chung Wei, Le-Yin Hsu, Shi-Heng Wang. Risk of acute mesenteric ischemia in patients with diabetes: A population-based cohort study in Taiwan. Atherosclerosis. Volume 296, P18-24, March 01,2020

9. Case report. T Todani, Y Sato, Y Watanabe, A Toki, S Uemura. Ischemic jejunal stricture developing after diabetic coma in a girl: a case report. Eur J Pediatar Surg. 1993 Apr;3(2):115-7

10. Dr Mohammad Taghi Niknejad and Assoc Prof Frank Gaillard. Mesentric ischemia. Radiopaedia. 